\title{
On the Ratio of the Energy Gap Amplitude to the Critical Temperature for Cuprates
}

\author{
R. SZCZĘ̧́SNIAK AND A.P. DuRAJSKI*
}

Institute of Physics, Częstochowa University of Technology, al. Armii Krajowej 19, 42-200 Częstochowa, Poland

\begin{abstract}
The model for the cuprates based on the modified electron-phonon pairing mechanism has been tested. For this purpose, the superconductors with high value of the critical temperature have been taken into consideration. In particular: $\mathrm{YBa}_{2} \mathrm{Cu}_{3} \mathrm{O}_{7-y}, \mathrm{HgBa}_{2} \mathrm{CuO}_{4+y}, \mathrm{HgBa}_{2} \mathrm{Cu}_{1-x} \mathrm{Zn}_{x} \mathrm{O}_{4+y}$, and $\mathrm{HgBa}_{2} \mathrm{Ca}_{2} \mathrm{Cu}_{3} \mathrm{O}_{8+y}$. It has been shown that the dependence of the ratio $R_{1} \equiv 2 \Delta_{\mathrm{tot}}^{(0)} / k_{\mathrm{B}} T_{\mathrm{C}}$ on the doping $(p)$ can be properly predicted in the framework of the presented theory; the symbol $\Delta_{\text {tot }}^{(0)}$ denotes the energy gap amplitude at the temperature of zero kelvin, and $T_{\mathrm{C}}$ is the critical temperature. The numerical results have been supplemented by the formula which describes the function $R_{1}(p)$.
\end{abstract}

DOI: 10.12693/APhysPolA.126.A-92

PACS/topics: 74.20.-z, 74.20.Fg, 74.20.Mn, 74.25.Bt, 74.72.-h, 74.72.Bk, 74.72.Jt

\section{Introduction}

In the solid state physics, the issue of the correctly determining the pairing mechanism in the hightemperature superconductors (cuprates) is very controversial [1]. Essentially, there is a clash of the two views. First of them is based on the original Fröhlich idea indicating that the interaction of the electron gas with the phonons is responsible for the formation of the superconducting state [2]. The second view favors the clean electron correlations, which in the simplest way can be modeled by the one-band Hubbard Hamiltonian [3].

It is true that in the cuprates, the classical electronphonon interaction is over one level weaker than the electron correlations [4]. Hence, it may seem that the limitation to the correlations in the electronic subsystem is correct. Unfortunately, in the framework of the Hubbard model with the positive values of the potential $\left(U_{\mathrm{H}}\right)$ it is very difficult to describe the thermodynamic properties of the high-temperature superconducting state. What is even worse, there is no convincing evidence that the superconducting state can exist at the sufficiently high temperature [5]. On the other hand, in the case of the negative value of the interaction potential with the same intensity of $U_{\mathrm{H}}$, proving the existence of the superconducting state with the high critical temperature is a simple matter.

Additionally, it is easy to prove that the pairing mechanism based on the classical electron-phonon interaction is insufficient to fully describe the superconducting state in the cuprates $[6,7]$.

\section{Model}

In the presented paper, we have tested the pairing mechanism, which combines two opposing approaches.

\footnotetext{
${ }^{*}$ corresponding author; e-mail: adurajski@wip.pcz.pl
}

The new mechanism has been proposed recently in [8] (see also [9] and [10]). It is based on three postulates: (i) In the superconductivity domain of the cuprates the fundamental role is played by the electrons on the $\mathrm{CuO}_{2}$ planes. (ii) In the cuprates there exists the conventional electron-phonon interaction, which does not have to be strong. (iii) In the cuprates there exist strong electronic correlations, but the electron-electron scattering in the superconductivity domain is inseparably connected with absorption or emission of the vibrational quanta.

By analyzing the set of the given postulates in the terms of the classical theory of the superconductivity $[11,12]$, it can be noticed that the first and second theory is based on the same philosophy. Namely, the physical system is constantly treated in a holistic manner, without the artificial division into the electron and phonon subsystem.

The Hamiltonian corresponding to the presented postulates has been derived in the paper [8]. Then, using the canonical transformation and the formalism of the thermodynamic Green functions one can get the equation determining the properties of the $d$-wave superconducting state. A detailed form of the equation is presented in Appendix.

The considered model has two main input parameters: the electron-phonon pairing potential $V^{(\eta)}$ and the electron-electron-phonon potential $U^{(\eta)}$. The first parameter can be calculated based on the value of the critical temperature $\left(T_{\mathrm{C}}\right)$, the second parameter has been chosen in such a way that it reproduces the value of the temperature at which the pseudogap appears $\left(T^{\star}\right)$. The values of two other input parameters (the hopping integral and the characteristic phonon frequency) have been taken from the literature.

On the basis of the calculated input parameters, we have determined the ratio of the energy gap amplitude at the temperature of zero kelvin to the critical temperature $\left(R_{1} \equiv 2 \Delta_{\text {tot }}^{(0)} / k_{\mathrm{B}} T_{\mathrm{C}}\right)$. The obtained results have been compared with the experimental 

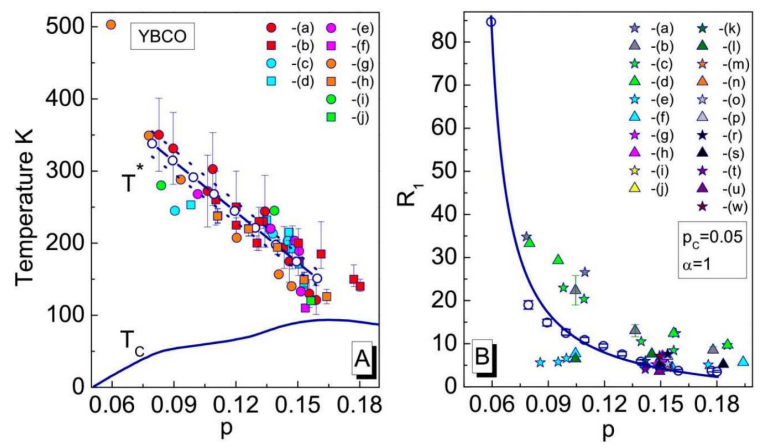

Fig. 1. (A) The temperatures $T_{\mathrm{C}}$ and $T^{\star}$ as the functions of the doping in YBCO. The empty circles represent the averaged values of $T^{\star}$. The filled circles and squares are the experimental data: (a) Kabanov et al. [13], (b) Daou et al. [14], (c) Solovjov et al. [15], (d) Obolenskii et al. [16], (e) Prokof'ev et al. [17], (f) Timusk et al. [18], (g) Chaban [19], (h) Vignolle et al. [20], (i) Lin et al. [21], (j) Rice et al. [22]. (B) The dependence of the ratio $R_{1}$ on the doping for YBCO. The empty circles represent the numerical results. The line has been prepared on the basis of the formula (1). The stars and the triangles are the experimental data: (a) Sutherland et al. [23], (b) Nakayama et al. [24], (c) Kaminski et al. [25], (d) Plate et al. [26], (e) Morr and Pines [27], Fong et al. [28], (f) Yeh et al. [29], (g) Born et al. [30], (h) Murakami et al. [31], (i) Edwards et al. [32], (j) Edwards et al. [33], (k) Tsai et al. [34], (l) Schrieffer and Brooks [35], (m) Maggio-Aprile et al. [36, 37], (n) Koinuma et al. [38], (o) Koyanagi et al. [39], (p) Kugler et al. [40], (r) Nantoh et al. [41, 42], (s) Shibata et al. [43], Ueno et al. [44, 45], (t) Kirtley et al. [46], (u) Hoevers et al. [47], (w) Tanaka et al. [48].
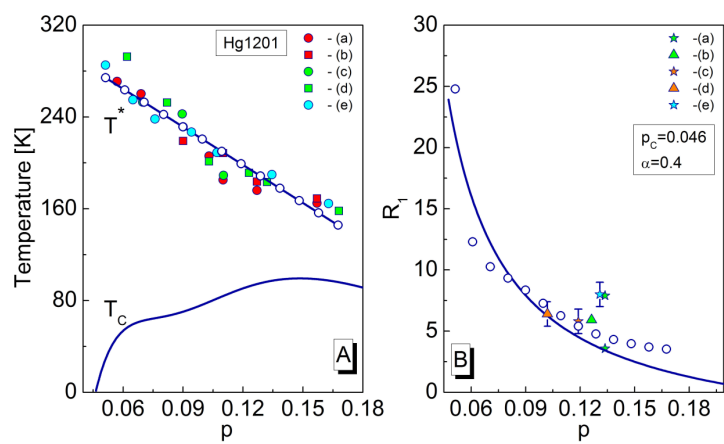

Fig. 2. (A) The temperatures $T_{\mathrm{C}}$ and $T^{\star}$ as the functions of the doping in $\mathrm{Hg} 1201$. The empty circles represent the averaged values of $T^{\star}$. The filled circles and squares are the experimental data: (a), (b) Yamamoto et al. [49], (c) Bandyopadhyay and Poddar [50], (d) Yamamoto et al. [51], (e) Honma et al. [52]. (B) The dependence of the ratio $R_{1}$ on the doping for $\mathrm{Hg} 1201$. The empty circles represent the strict numerical results. The line has been prepared on the basis of the formula (1). The stars and the triangles are the experimental data: (a) Wei et al. [53], (b) Hasegawa et al. [54], (c) Yang et al. [55], (d) Guyard et al. [56], (e) Inosov et al. [57]. data. In particular, the following compounds have been studied: $\mathrm{YBa}_{2} \mathrm{Cu}_{3} \mathrm{O}_{7-y}$ (YBCO), $\mathrm{HgBa}_{2} \mathrm{CuO}_{4+y}$ (Hg1201), $\quad \mathrm{HgBa}_{2} \mathrm{Cu}_{1-x} \mathrm{Zn}_{x} \mathrm{O}_{4+y} \quad(\mathrm{Hg} 1201-\mathrm{Zn}), \quad$ and $\mathrm{HgBa}_{2} \mathrm{Ca}_{2} \mathrm{Cu}_{3} \mathrm{O}_{8+y}$ (Hg1223). It should be noted that the considered compounds are the examples of the superconductors with the highest critical temperature (the optimal doping).
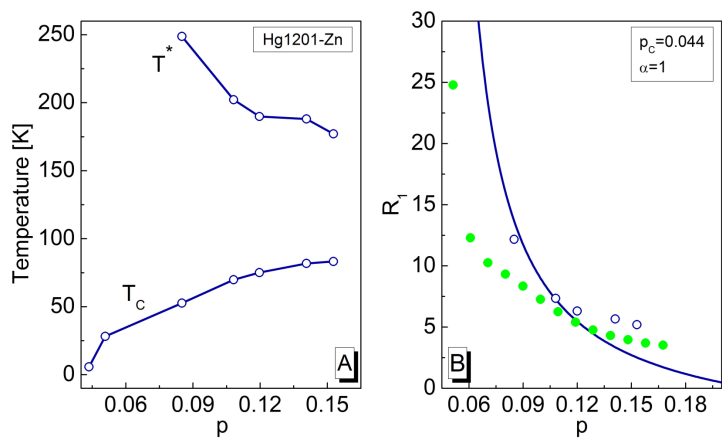

Fig. 3. (A) The temperatures $T_{\mathrm{C}}$ and $T^{\star}$ as the functions of the doping in Hg1201-Zn. The diagram has been made on the basis of the paper by Yamamoto et al. [51]. (B) The dependence of the ratio $R_{1}$ on the doping. The empty circles represent the strict numerical results for $\mathrm{Hg} 1201-\mathrm{Zn}$. The line has been prepared on the basis of the formula (1). The green filled circles show the results achieved for $\mathrm{Hg} 1201$.
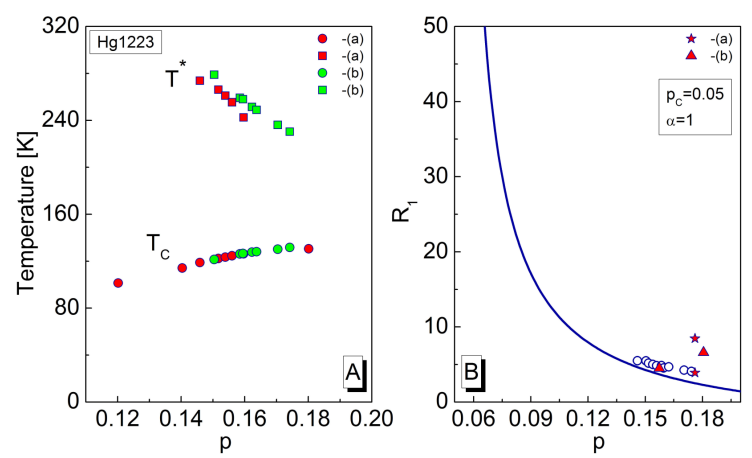

Fig. 4. (A) The temperatures $T_{\mathrm{C}}$ and $T^{\star}$ as the functions of the doping in $\mathrm{Hg} 1223$. The diagram has been formed on the basis of the following experimental data: (a) Liu et al. [58] and (b) Lam and Vyas [59]. (B) The dependence of the ratio $R_{1}$ on the doping for $\mathrm{Hg} 1223$. The empty circles represent the strict numerical results. The line has been prepared on the basis of the formula (1). The experimental data: (a) Jeong et al. [60], (b) Rossel et al. [61].

\section{Results}

In the first step, the ratio $R_{1}$ for the superconductor YBCO has been calculated. In Fig. 1A there are presented the experimental courses of $T_{\mathrm{C}}(p)$ and $T^{\star}(p)$, where $p$ denotes the concentration of the holes (the doping). The function $T_{\mathrm{C}}(p)$ has been plotted on the basis 
of the data included in the paper [62]; the values $T^{\star}(p)$ have been determined averaging the large number of the experimental data. Then, on the basis of the equation (A1), the pairing potentials $V^{(\eta)}$ and $U^{(\eta)}$ have been calculated.

Next, we have prepared the dependence $R_{1}(p)$ (Fig. 1B). It has been found that the theoretical results correctly reproduce the experimental data.

Let us notice that the dependence of the critical temperature on the doping for the cuprates has been parameterized with the help of the expression: $T_{\mathrm{C}}(p) / T_{\mathrm{C}, \max }=$ $1-82.6(p-0.16)^{2}$ [63]. The shape of the function $R_{1}(p)$ can be reproduced in a similar way. In the considered case, the following result has been obtained:

$$
R_{1}(p)=\frac{p-5 p_{c}}{\alpha p_{c}-p}\left[R_{1}\right]_{\mathrm{dBCS}},
$$

where $p_{c}$ is the value of the concentration of the holes at which the superconducting state appears; $\alpha$ is the fitting parameter. For YBCO, it has been assumed that: $p_{c}=0.05$ and $\alpha=1$. The symbol $\left[R_{1}\right]_{\mathrm{dBCS}}$ denotes the value of the ratio $R_{1}$ calculated in the framework of the $d$-wave BCS model: $\left[R_{1}\right]_{\mathrm{dBCS}}=4.28[64]$. The form of the analytical results has been presented in Fig. 1B.

In the same way the course of the dependence of $R_{1}$ on $p$ in the family of the high temperature superconductors containing mercury has been submitted. The results have been presented in Figs. 2-4.

In the case of the superconductor $\mathrm{Hg} 1201$, the shape of the function $T_{\mathrm{C}}(p)$ has been obtained on the basis of the papers $[49,51$, and 65$]$; the averaged values of $T^{\star}(p)$ have been determined with the help of the experimental data presented in Fig. 2A. The theoretical results and the experimental data have been collected in Fig. 2B. It has been found that the theoretical predictions agree with the experimental data. Then, by using the formula (1), the course of the function $R_{1}(p)$ has been reproduced; the following parameterization has been selected: $p_{c}=$ 0.046 and $\alpha=0.4$. Also in this case the formula (1) correctly reproduces the dependence of the ratio $R_{1}$ on the concentration of the holes.

The superconductor $\mathrm{Hg} 1201-\mathrm{Zn}$ represents the case for which no experimental values of $R_{1}(p)$ have been found in the literature. However, there are well defined functions $T_{\mathrm{C}}(p)$ and $T^{\star}(p)$ (Fig. 3A). Thus, the course of the dependence $R_{1}(p)$ can be calculated theoretically. The strict numerical data have been collected in Fig. 3B. In addition, there have been presented the results obtained on the basis of the formula (1), for which it has been assumed that $p_{c}=0.044$ and $\alpha=1$.

It is easy to notice that for $p \geq 0.11$, in the relation to the course obtained for $\mathrm{Hg} 1201$, the disorder induced by zinc does not exert the significant influence on $R_{1}(p)$. Much bigger deviations can be noticed for the lower values of the doping. The discussed effect can be relatively easily verified by the experimenters — we strongly encourage to do so.

The superconductor Hg1223 is characterized with the highest observed critical temperature for the optimal doping $\left(T_{\mathrm{C}} \approx 135 \mathrm{~K}\right)[66]$. The full courses of $T_{\mathrm{C}}(p)$ and $T^{\star}(p)$ for $\operatorname{Hg} 1223$ have been determined in the paper [58] and [59] (Fig. 4A). Using the already discussed method of the analysis, we have obtained the theoretical form of the function $R_{1}(p)$. The results have been presented in Fig. 4B. We have found that the theoretical predictions reproduce the existing experimental data very well. Additionally, in Fig. 4B there have been plotted the analytical values of $R_{1}(p)$; we have assumed that $p_{c}=0.05$ and $\alpha=1$.

\section{Summary}

In the presented paper, we have calculated the dependence of the ratio $R_{1}$ on the doping for four superconductors characterized by high values of the critical temperature. In the case of YBCO, Hg1201 and Hg1223, the theoretical results reproduce the experimental data very well. For the superconductor Hg1201-Zn only the theoretical results have been provided due to the fact that the relevant experiments have not been undertaken yet. Finally, we have shown that the shape of the function $R_{1}(p)$ can be reproduced by the analytical formula.

The presented theory can be used to explore all other thermodynamic parameters of the cuprates. It seems that one of the most fundamental issues is associated with the interpretation of the experimental data obtained by using the ARPES method. Such studies are currently underway and, in a short time, the results will be presented.

\section{Appendix: the fundamental equation}

The equation for the high temperature superconducting state has the form [8] (see also [9] and [10]):

$$
\begin{aligned}
1 & =\left(V^{(\eta)}+\frac{U^{(\eta)}}{6}\left|\Delta^{(\eta)}\right|^{2}\right) \\
& \times \frac{1}{N_{0}} \sum_{\boldsymbol{k}}^{\omega_{0}} \frac{\eta^{2}(\boldsymbol{k})}{2 E_{\boldsymbol{k}}^{(\eta)}} \tanh \frac{\beta E_{\boldsymbol{k}}^{(\eta)}}{2},
\end{aligned}
$$

where the quantities $V^{(\eta)}$ and $U^{(\eta)}$ denote the effective pairing potentials for the electron-phonon and electronelectron-phonon interaction, respectively. The symbol $\Delta^{(\eta)}$ is the amplitude of the $d$-wave order parameter; $\eta(\boldsymbol{k}) \equiv 2\left[\cos \left(k_{x}\right)-\cos \left(k_{y}\right)\right]$. The function $E_{\boldsymbol{k}}^{(\eta)}$ is given by the expression

$$
\begin{aligned}
& E_{\boldsymbol{k}}^{(\eta)} \equiv \\
& \quad \sqrt{\varepsilon_{\boldsymbol{k}}^{2}+\left(V^{(\eta)}+\frac{U^{(\eta)}}{6}\left|\Delta^{(\eta)}\right|^{2}\right)^{2}\left(\left|\Delta^{(\eta)}\right| \eta(\boldsymbol{k})\right)^{2}},
\end{aligned}
$$

where $\varepsilon_{\boldsymbol{k}}$ denotes the electron band energy: $\varepsilon_{\boldsymbol{k}}=$ $-t \gamma(\boldsymbol{k}) ; \quad t$ is the hopping integral and $\gamma(\boldsymbol{k}) \equiv$ $2\left[\cos \left(k_{x}\right)+\cos \left(k_{y}\right)\right]$. The quantity $\beta$ has been defined as: $\beta \equiv 1 / k_{\mathrm{B}} T$, where $k_{\mathrm{B}}$ is the Boltzmann constant.

Equation (A1) has too much a complicated form to be solved in the analytical way. For this reason, 
it has been analyzed by using the numerical methods. Additionally, we have assumed that: $\sum_{k}^{\omega_{0}} \simeq$ $\int_{-\pi}^{\pi} \int_{-\pi}^{\pi} \mathrm{d} k_{x} \mathrm{~d} k_{y} \theta\left(\omega_{0}-\left|\varepsilon_{\left(\boldsymbol{k}_{x}, \boldsymbol{k}_{y}\right)}\right|\right)$, where $\theta$ is the Heaviside function. The normalization constant is given by the following: $N_{0} \equiv 1 / \sum_{k}^{\omega_{0}}$; the symbol $\omega_{0}$ represents the characteristic phonon frequency, which is of the order of the Debye frequency.

The energy gap amplitude at the temperature of zero kelvin is defined as: $\Delta_{\text {tot }}^{(0)} \equiv\left(V^{(\eta)}+\frac{U^{(\eta)}}{6}\left|\Delta_{0}^{(\eta)}\right|^{2}\right)\left|\Delta_{0}^{(\eta)}\right|$.

\section{References}

[1] J.G. Bednorz, K.A. Müller, Z. Phys. B 64, 189 (1986).

[2] H. Fröhlich, Phys. Rev. 79, 845 (1950).

[3] J. Hubbard, Proc. R. Soc. London Ser. A 276, 238 (1963).

[4] J.P. Hauge, AIP Conf. Proc. 846, 255 (2006).

[5] D.J. Scalapino, S.R. White, S.C. Zhang, Phys. Rev. Lett. 68, 2830 (1992).

[6] R. Szczȩśniak, M. Mierzejewski, J. Zieliński, P. Entel, Solid State Commun. 117, 369 (2001).

[7] R. Szczȩśniak, Solid State Commun. 138, 347 (2006).

[8] R. Szczȩśniak, PloS ONE 7, e31873 (2012).

[9] R. Szczȩśniak, arXiv:1105.5525 (2011).

[10] R. Szczȩśniak, arXiv:1110.3404 (2012).

[11] J. Bardeen, L.N. Cooper, J.R. Schrieffer, Phys. Rev. 106, 162 (1957).

[12] J. Bardeen, L.N. Cooper, J.R. Schrieffer, Phys. Rev. 108, 1175 (1957).

[13] V.V. Kabanov, J. Demsar, B. Podobnik, D. Mihailovic, Phys. Rev. B 59, 1497 (1999).

[14] R. Daou, J. Chang, D. LeBoeuf, O. Cyr-Choiniere, F. Laliberte, N. Doiron-Leyraud, B.J. Ramshaw, R. Liang, D.A. Bonn, W.N. Hardy, L. Taillefer, $\mathrm{Na}$ ture 463, 519 (2010).

[15] A.L. Solovév, S.L. Sidorov, V.Y. Tarenkov, A.I. Dýachenko, Low Temp. Phys. $\mathbf{3 5}, 826$ (2009).

[16] M.A. Obolenskii, R.V. Vovk, A.V. Bondarenko, N.N. Chebotaev, Low Temp. Phys. 32, 571 (2006).

[17] D. Prokofév, M. Volkov, Y. Boikov, Phys. Solid State 45, 1223 (2003).

[18] T. Timusk, B. Statt, Rep. Prog. Phys. 62, 61 (1999).

[19] I. Chaban, Phys. Solid State 50, 803 (2008).

[20] B. Vignolle, B.J. Ramshaw, J. Day, D. LeBoeuf, S. Lepault, R. Liang, W.N. Hardy, D.A. Bonn, L. Taillefer, C. Proust, Phys. Rev. B 85, 224524 (2012).

[21] J.-Y. Lin, P.R. Lee, Y.T. Liu, Ch.-Y. Mou, Y.-J. Chen, K.H. Wu, C.W. Luo, J.Y. Juang, T.M. Uen, J.M. Lee, J.M. Chen, arXiv:1009.2560v2 (2010).

[22] J.P. Rice, J. Giapintzakis, D.M. Ginsberg, J.M. Mochel, Phys. Rev. B 44, 10158 (1991).
[23] M. Sutherland, D.G. Hawthorn, R.W. Hill, F. Ronning, S. Wakimoto, H. Zhang, C. Proust, E. Boaknin, C. Lupien, L. Taillefer, R. Liang, D.A. Bonn, W.N. Hardy, R. Gagnon, N.E. Hussey, T. Kimura, M. Nohara, H. Takagi, Phys. Rev. B 67, 174520 (2003).

[24] K. Nakayama, T. Sato, K. Terashima, T. Arakane, T. Takahashi, M. Kubota, K. Ono, T. Nishizaki, Y. Takahashi, N. Kobayashi, Phys. Rev. B $\mathbf{7 9}$, 140503(R) (2009).

[25] A. Kaminski, S. Rosenkranz, H.M. Fretwell, J. Mesot, M. Randeria, J.C. Campuzano, M.R. Norman, Z.Z. Li, H. Raffy, T. Sato, T. Takahashi, K. Kadowaki, Phys. Rev. B 69, 212509 (2004).

[26] M. Plate, J.D.F. Mottershead, I.S. Elfimov, D.C. Peets, R. Liang, D.A. Bonn, W.N. Hardy, S. Chiuzbaian, M. Falub, M. Shi, L. Patthey, A. Damascelli, Phys. Rev. Lett. 95, 077001 (2005).

[27] D.K. Morr, D. Pines, Phys. Rev. Lett. 81, 1086 (1998).

[28] H.F. Fong, B. Keimer, D.L. Milius, I.A. Aksay, Phys. Rev. Lett. 78, 713 (1997).

[29] N.-C. Yeh, C.-T. Chen, G. Hammerl, J. Mannhart, A. Schmehl, C.W. Schneider, R.R. Schulz, S. Tajima, K. Yoshida, D. Garrigus, M. Strasik, Phys. Rev. Lett. 87, 087003 (2001).

[30] V. Born, C. Jooss, H.C. Freyhardt, Physica C 382 , 224 (2002).

[31] H. Murakami, H. Asaoka, K. Sakai, T. Ito, M. Tonouchi, Appl. Surf. Sci. 175-176, 306 (2001).

[32] H.L. Edwards, J.T. Markert, A.L. de Lozanne, Phys. Rev. Lett. 69, 2967 (1992).

[33] H.L. Edwards, D.J. Derro, A.L. Barr, J.T. Markert, A.L. de Lozanne, Phys. Rev. Lett. 75, 1387 (1995).

[34] J.S. Tsai, I. Takeuchi, J. Fujita, S. Miura, T. Terashima, Y. Bando, K. Iijima, K. Yamamoto, Physica C 157, 537 (1989).

[35] J.R. Schrieffer, J.S. Brooks, Handbook of HighTemperature Superconductivity. Theory and Experiment, Springer, Berlin 2007.

[36] I. Maggio-Aprile, Ch. Renner, A. Erb, E. Walker, Ø. Fischer, Phys. Rev. Lett. 75, 2754 (1995).

[37] I. Maggio-Aprile, Ch. Rennet, A. Erb, E. Walker, Ø. Fischer, Nature 390, 487 (1997).

[38] H. Koinuma, S. Gonda, J.P. Gong, M. Kawasaki, M. Yoshimoto, M. Nantoh, T. Hasegawa, K. Kitazawa, J. Phys. Chem. Solids 54, 1215 (1993).

[39] M. Koyanagi, S. Kashiwaya, M. Matsuda, H. Takashima, K. Kajimura, Jpn. J. Appl. Phys. 34, 89 (1995).

[40] M. Kugler, Ch. Renner, Ø. Fischer, V. Mikheev, G. Batey, Rev. Sci. Instrum. 71, 1475 (2000).

[41] M. Nantoh, T. Hasegawa, M. Kawasaki, J.P. Gong, K. Fujito, A. Takagi, W. Yamaguchi, M. Ogino, H. Koinuma, K. Kitazawa, J. Supercond. 7, 349 (1994).

[42] M. Nantoh, M. Kawasaki, T. Hasegawa, K. Fujito, W. Yamaguchi, H. Koinuma, K. Kitazawa, Physica C 242, 277 (1995).

[43] K. Shibata, M. Maki, T. Nishizaki, N. Kobayashi, Physica C 388-389, 277 (2003). 
[44] S. Ueno, S. Kashiwaya, N. Terada, A. Sawa, N. Matsubara, M. Koyanagi, Y. Tanaka, Y. Tanuma, K. Obara, K. Takita, Physica C 357-360, 1576 (2001).

[45] S. Ueno, S. Kashiwaya, N. Terada, Y. Tanaka, Y. Tanuma, M. Koyanagi, K. Obara, Physica C 388389, 301 (2003).

[46] J.R. Kirtley, R.T. Collins, Z. Schlesinger, W.J. Gallagher, R.L. Sandstrom, T.R. Dinger, D.A. Chance, Phys. Rev. B 35, 8846 (1987).

[47] H.F.C. Hoevers, P.J.M. Van Bentum, L.E.C. Van De Leemput, H. Van Kempen, A.J.G. Schellingerhout, D. Van Der Marel, Physica C 152, 105 (1988).

[48] S. Tanaka, E. Ueda, M. Sato, Physica C 224, 126 (1994).

[49] A. Yamamoto, W.Z. Hu, S. Tajima, Phys. Rev. B 63, 024504 (2000).

[50] B. Bandyopadhyay, A. Poddar, J. Alloys Comp. 326, 137 (2001).

[51] A. Yamamoto, K. Minami, W.-Z. Hu, A. Miyakita, M. Izumi, S. Tajima, Phys. Rev. B 65, 104505 (2002).

[52] T. Honma, P.H. Hor, H.H. Hsieh, M. Tanimoto, Phys Rev. B 70, 214517 (2004).

[53] J.Y.T. Wei, C.C. Tsuei, P.J.M. van Bentum, Q. Xiong, C.W. Chu, M.K. Wu, Phys. Rev. B 57, 3650 (1998).

[54] T. Hasegawa, H. Ikutaand, K. Kitazawa, Physical Properties of High Temperature Superconductors III, World Sci., 1992.
[55] J. Yang, J. Hwang, E. Schachinger, J.P. Carbotte, R.P.S.M. Lobo, D. Colson, A. Forget, T. Timusk, Phys. Rev. Lett. 102, 027003 (2009).

[56] W. Guyard, A. Sacuto, M. Cazayous, Y. Gallais, M. Le Tacon, D. Colson, A. Forget, Phys. Rev. Lett. 101, 097003 (2008).

[57] D.S. Inosov, J.T. Park, A. Charnukha, Y. Li, A.V. Boris, B. Keimer, V. Hinkov, Phys. Rev. B 83, 214520 (2011)

[58] S. Liu, G. Wu, X. Xu, H. Shao, J. Supercond. 17, 253 (2004).

[59] C.C. Lam, A. Vyas, Int. J. Mod. Phys. B 15, 2285 (2001).

[60] G.T. Jeong, J.I. Kye, S.H. Chun, S. Lee, S.I. Lee, Z.G. Khim, Phys. Rev. B 49, 15416 (1994).

[61] C. Rossel, R.R. Schulz, A. Schilling, H.R. Ott, J. Karpinski, Physica C 235-240, 1871 (1994)

[62] R. Liang, D.A. Bonn, W.N. Hardy, Phys. Rev. B 73, 180505(R) (2006)

[63] M.R. Presland, J.L. Tallon, R.G. Buckley, R.S. Liu, N.E. Flower, Physica C 176, 95 (1991).

[64] H. Won, K. Maki, Phys. Rev. B 49, 1397 (1994).

[65] H.G. Luo, Y.H. Su, T. Xiang, Phys. Rev. B 77, 014529 (2008).

[66] C.W. Chu, L. Gao, F. Chen, Z.J. Huang, R.L. Meng, Y.Y. Xue, Nature 365, 323 (1993). 